\title{
Active surveillance comes of age
}

A prospective study of active surveillance in 993 men with favourable-risk prostate cancer has yielded long-term results that further support its safety.

Active surveillance can reduce overtreatment of indolent disease while retaining the option of treatment in response to cancer progression, but requires long-term validation and comparison of outcomes with those of initial definitive therapy.

Klotz et al. enrolled patients with low-risk (Gleason score $\leq 6$, PSA $\leq 10 \mathrm{ng} / \mathrm{ml}$ ) or favourable, intermediaterisk (including Gleason score $3+4$, PSA $10-20 \mathrm{ng} / \mathrm{ml}$ ) disease. Patients were assessed with regular PSA measurements and prostate biopsy, and intervention was offered for PSA-doubling time $<3$ years, Gleason score progression or unequivocal clinical progression.

The study was initiated in 1995, and since then, 149 participants have died, and 819 have survived and been treated within the study protocol, with a median follow-up time from first biopsy of 6.4 years (range $0.2-19.8$ years). 15 deaths from prostate cancer have been recorded, all involving metastatic disease. Metastasis also occurred in a further 13 men, four of whom died from other causes, and nine of whom were still alive at the time of data lock (May 2013). The cumulative hazard ratio for other cause mortality relative to prostate cancer mortality was 9.2:1.0.

267 patients have so far received additional prostate cancer therapy for disease progression, whereas 75.7\%, 63.5\% and $55.0 \%$ of men observed for 5, 10 and 15 years, respectively, have remained untreated. Active surveillance is already a valid approach for selected patients, and future developments in prognostic ability can refine eligibility criteria and minimize deaths caused by deferral of treatment.

Robert Phillips

Original article Klotz, L. et al. Long-term follow-up of a large active surveillance cohort of patients with prostate cancer. J. Clin. Oncol. doi:10.1200/JC0.2014.55.1192 